\title{
Solidified Floating Organic Drop Microextraction for the Determination of Trace Dy and $Y$ by Electrothermal Vaporization Inductively Coupled Plasma Mass Spectrometry
}

\author{
Shizhong Chen*, Shengping Zhu, and Dengbo Lu \\ College of Chemical and Environmental Engineering, Wuhan Polytechnic University, \\ Wuhan 430023, P.R. China
}

\section{INTRODUCTION}

Rare earth elements (REEs) have been widely used in modern industry and agriculture owing to their specific properties $(1,2)$. As a result, more and more REEs are entering the environment. An investigation showed that continuous exposure to low concentrations of REEs could cause adverse health effects because of their bioaccumulation along the food chain (3). Chronic exposure to REE dust probably causes occupational pneumoconiosis (4). Therefore, the determination of trace REE concentrations in various matrices has attracted considerable interest.

It is well known that inductively coupled plasma mass spectrometry (ICP-MS) is one of the most effective tools used for the determination of trace REEs owing to its high sensitivity, wide linear dynamic range, rapid multielement detection capability, and absence of line-rich spectrum interferences encountered in inductively coupled plasma atomic emission spectrometry. However, direct determination of REEs in biological and environmental samples by ICP-MS often suffers from extremely low REE concentrations and complex matrix effects. To achieve an accurate and reliable result, a chemical separation and preconcentration step is often required prior to their determination. A variety of methods, including solvent extraction, coprecipi-

\footnotetext{
*Corresponding author.

E-mail: chenshizhong62@163.com

Fax: +862783956442
}

\begin{abstract}
A method was developed for the determination of trace Dy and $\mathrm{Y}$ in biological and environmental water samples based on solidified floating organic drop microextraction (SFODME) combined with electrothermal vaporization (ETV) inductively coupled plasma mass spectrometry (ICPMS). 1-(2-pyridylazo)-2-naphthol (PAN) was used as both chelating reagent in SFODME and chemical modifier in ETV for separation, preconcentration, and volatilization of the REEs prior to their determination. The main factors affecting the preconcentration and determination, including $\mathrm{pH}$, amount of PAN, extraction time, stirring rate, sample solution volume, and temperature program, were examined in detail.

Under the selected conditions, the detection limits of this method were $0.019 \mathrm{pg} \mathrm{mL}^{-1}$ of Dy and $0.032 \mathrm{pg} \mathrm{mL}^{-1}$ of $\mathrm{Y}$, and the relative standard deviations were $4.7 \%$ and $5.4 \%$, respectively $\left(\mathrm{n}=9, \mathrm{c}=0.1 \mathrm{ng} \mathrm{mL}^{-1}\right)$. This method was applied for the determination of trace Dy and Y in certified reference material and natural water samples with recoveries of $91.3-108 \%$.
\end{abstract}

tation, ion exchange, solid phase extraction, chelating resin adsorption, and cloud point extraction have been commonly used for sample pretreatment (5-9).

Although relatively good analytical performances can be obtained with these techniques, they have some disadvantages, such as large consumption of reagent, multistage operation, unsatisfactory enrichment factor, and lengthy separation. To simplify the pretreatment process, reduce reagent consumption, and avoid damaging human health, a series of miniaturized sample pretreatment procedures have been developed in the past decades. Of them, liquid phase microextraction (LPME) has attracted considerable interest in analytical chemistry since it was reported in the mid-to-late 1990 s $(10,11)$. LPME has different extraction modes, including single-drop microextraction, hollow fiber liquid phase microextraction, and dispersive liquid-liquid microextraction (10, 12-14). However, these still have some inherent drawbacks, such as instability of droplet, formation of air bubbles, and use of toxic reagents in the sample pretreatment process.

In order to overcome the problems mentioned above, a new LPME technique, namely solidified floating organic drop microextraction (SFODME), was proposed for extraction and determination of organic analytes (15). In this technique, a free microdrop of organic solvent with a melting point near room temperature (in the range of $10-30^{\circ} \mathrm{C}$ ) is floated on the surface of the aqueous solution. The aqueous phase is stirred for a prescribed period of time, and then the sample vial is transferred into an ice bath. After the organic solvent is solidified, it is transferred into a small conical vial, where it melts quickly at room temperature. Finally, it is retracted by a microsyringe and 
injected into an instrument for subsequent analysis. Owing to its unique advantages of simplicity, speed, low cost, small consumption of organic solvent, and high enrichment factor, SFODME has been successfully employed for the determination of trace elements in various samples (16-20). However, to the best of our knowledge, the use of SFODME for preconcentration, separation, and determination of REEs has received little attention so far.

Electrothermal vaporization (ETV), as a sample introduction device for ICP-MS, has the merits of high introduction efficiency and small sample requirement. As described previously, SFODME is a miniaturized sample pretreatment technique, and ETV is a microamount sample introduction device. Therefore, SFODME coupled with ETV for ICP-MS may be a perfect combination. The aim of this work was to use 1-(2-pyridylazo)-2-naphthol (PAN) as both a chelating reagent in SFODME and a chemical modifier in ETV for separation, preconcentration, and volatilization of REEs prior to their determination by ICP-MS. Based on the obtained experimental results, a method of SFODME-ETV-ICP-MS was developed for the determination of trace Dy and Y in biological and environmental samples with satisfactory results.

\section{EXPERIMENTAL}

\section{Instrumentation}

An X-7 ICP-MS system (Thermo Elemental Corporation, USA), equipped with a modified commercially available WF-4C graphite furnace (Beijing Second Optics, P.R. China) as an electrothermal vaporizer, was used for determination of the analytes. The operating parameters of ICP-MS were optimized with a concentric glass nebulizer prior to connection with the ETV device. A pyrolytic graphite tube was used throughout this work. The working conditions of ETV-ICP-MS are summarized in Table I.

The $\mathrm{pH}$ value of the solution was controlled with a $\mathrm{pH}$ meter (Thermo Orion Corporation, USA) supplied with a combined electrode. An Ethos $\mathrm{T}$ microwave digestion device (Milestone, Italy) was used for sample digestion.

\section{Standard Solutions and Reagents}

Stock standard solutions of Dy and $\mathrm{Y}$ at $1.0 \mathrm{mg} \mathrm{mL}^{-1}$ were obtained from the National Analysis Center of Iron \& Steel (Beijing, P.R. China). All reagents used in this study were of high purity or at least of analytical grade. Standard solutions of Dy and $\mathrm{Y}$ were prepared daily by appropriate dilution of their stock solutions. The extraction solvent 1-undecanol was used (Shanghai Reagent Factory, P.R. China). PAN solution was prepared by dissolving proper amounts of PAN (Shanghai

Operating Parameters for ETV-ICP-MS

\begin{tabular}{|c|c|}
\hline \multicolumn{2}{|l|}{ ICP-MS Plasma } \\
\hline Plasma Power & $1300 \mathrm{~W}$ \\
\hline Plasma Argon Flow Rate & $14.5 \mathrm{~L} \mathrm{~min}^{-1}$ \\
\hline Auxiliary Argon Flow Rate & $0.85 \mathrm{~L} \mathrm{~min}^{-1}$ \\
\hline Nebulizer Argon Flow Rate & $0.82 \mathrm{~L} \mathrm{~min}^{-1}$ \\
\hline Sampler Orifice (nickel) & $1.1 \mathrm{~mm}$ \\
\hline Skimmer Orifice (nickel) & $0.7 \mathrm{~mm}$ \\
\hline Acquisition Mode & Peak jumping \\
\hline Number of Sweep & 100 \\
\hline Dwell Time & $10 \mathrm{~ms}$ \\
\hline Acquisition Time & $40 \mathrm{~s}$ \\
\hline Number of Measurements per Peak & 3 \\
\hline Isotopes & ${ }^{163}$ Dy and ${ }^{89} \mathrm{Y}$ \\
\hline \multicolumn{2}{|l|}{ ETV } \\
\hline Sample Volume & $10 \mu \mathrm{L}$ \\
\hline Carrier Gas Flow Rate & $0.45 \mathrm{~L} \mathrm{~min}^{-1}$ \\
\hline Drying Step & $100^{\circ} \mathrm{C}$, ramp $10 \mathrm{~s}$, hold $10 \mathrm{~s}$ \\
\hline Ashing Step & $220^{\circ} \mathrm{C}$, ramp $10 \mathrm{~s}$, hold $20 \mathrm{~s}$ \\
\hline Vaporization Step & $1200^{\circ} \mathrm{C}$, hold $4 \mathrm{~s}$ \\
\hline Clear-out Temperature & $2500^{\circ} \mathrm{C}$ \\
\hline
\end{tabular}

Reagent Factory, P.R. China) in 1-undecanol. All glass and polypropylene ware was kept in $2.0 \mathrm{~mol} \mathrm{~L}^{-1}$ $\mathrm{HNO}_{3}$ solutions for at least one night, and then rinsed with $0.1 \mathrm{~mol} \mathrm{~L}^{-1}$ $\mathrm{HNO}_{3}$ solution and doubly distilled water before use. High purity deionized water, obtained from a Milli-Q ${ }^{\mathrm{TM}}$ A10 system (Millipore Corporation, USA), was used throughout this work.

\section{Sample Preparation}

A portion $(0.1000 \mathrm{~g})$ of sample (tea leaves obtained from Zhushan, P.R. China) was accurately weighed into a $100-\mathrm{mL}$ PTFE vessel to which $4.0 \mathrm{~mL}$ of concentrated $\mathrm{HNO}_{3}$ and $2.0 \mathrm{~mL}$ of $\mathrm{H}_{2} \mathrm{O}_{2}$ were added. The sample was heated in the microwave oven at $180{ }^{\circ} \mathrm{C}$ (ramp $10 \mathrm{~min}$ utes; hold 15 minutes) with $1.0 \mathrm{~kW}$ power. After heating, the sample solution was cooled to room temperature. The obtained solution was transferred into a 50-mL PTFE beaker, and the vessel rinsed with

\section{.}

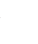


about $20 \mathrm{~mL}$ of deionized water, which was added to the beaker. The solution was then heated to near dryness. The residues were dissolved in $0.1 \mathrm{~mol} \mathrm{~L}^{-1} \mathrm{HNO}_{3}$ solution, and then diluted to the desired volume with deionized water.

A natural water sample (collected from the Hanjiang River in Wuhan) was filtered through a $0.45 \mu \mathrm{m}$ membrane filter and analyzed as soon as possible after sampling. The blank was prepared in exactly the same way as the sample.

\section{SFODME Extraction}

The SFODME device for REE extraction was similar to that described in the literature (15). The sample solution containing REE ions was adjusted to $\mathrm{pH} 7.0$ using diluted nitric acid or ammonium hydroxide. Then $10 \mathrm{~mL}$ of the resulting solution was transferred into an 11-mL vial, and a stir bar and $20 \mu \mathrm{L}$ of PAN in 1-dodecanol were added. The magnetic stirrer was turned on, and the solution stirred for a fixed time. In this step, REE ions react with PAN to form hydrophobic REE-PAN complexes, which were then extracted into 1-undecanol. After the extraction process was carried out, the sample vial was transferred into an ice bath until the organic solvent solidified. The solidified solvent was then transferred into a conical vial where it melted immediately at room temperature. The extract was diluted to $100 \mu \mathrm{L}$ with tetrahydrofuran (THF). Finally, $10 \mu \mathrm{L}$ of the extract was injected into the graphite tube for the determinations.

\section{ETV-ICP-MS Procedure}

After the ETV unit had been connected to the ICP-MS, $10 \mu \mathrm{L}$ of the analytes was injected into the graphite furnace. During the drying and charring process, the dosing hole of the graphite furnace was kept open to remove the water and organic vapor. It was then sealed with a graphite probe for 5 to 10 seconds prior to the vaporization step. The vaporized analytes were swept into the plasma excitation source by a carrier gas, and the peak hop transient mode for data acquisition was used for detection of the analytes.

\section{RESULTS AND DISCUSSION}

\section{Vaporization Behavior of REE-PAN Chelate}

The effects of PAN and 1-dodecanol on the vaporization characteristics of the REEs were investigated under the optimum conditions. In order to facilitate removal of the organic solvent without residues of the analytes in the sampler, THF was added into the extract obtained after separation from the sample solution. The typical signal profiles of $Y$ are shown in Figure 1. It can be seen that an intense and sharp signal was detected with PAN as the chemical modifier due to the quantitative extraction and vaporization of the volatile Y-PAN complex (B). On the contrary, no signal was recorded for the same concentration of a $\mathrm{Y}$ aqueous standard solution in the absence of PAN (C). A blank signal was not found in the experiment (A). A similar result was also observed for Dy. These results showed that in SFODME-ETV-ICP-

\section{Atomic Apectroscopy 1 Vol. 35(1), Jan./Feb. 2014}

MS, a REE-PAN complex can be formed rapidly, extracted effectively by 1-dodecanol, and vaporized quantitatively into the ICP.

\section{Choice of Chelating Reagent and Extraction Solvent}

The extraction solvent and chelating reagent are two factors for achieving high extraction efficiency and a stable complex. The extraction solvent has the merits of high extraction efficiency, low volatility, low water solubility, suitable melting point (about $10-30^{\circ} \mathrm{C}$ ), appropriate viscosity, and relatively low boiling point, which facilitate the extraction of analytes, solidification, and transfer of solvent, and $i n$ situ removal of the organic solvent in the subsequent ETV process. On the other hand, the selected chelating reagent should react rapidly with the analytes to form a thermally stable and volatile complex which can be extracted effectively and vaporized quantitatively into the ICP. According to our previous work (21) and the literature (15), PAN and 1-dodecanol were used as both the chelating reagent and the extraction solvent in this work, respectively.

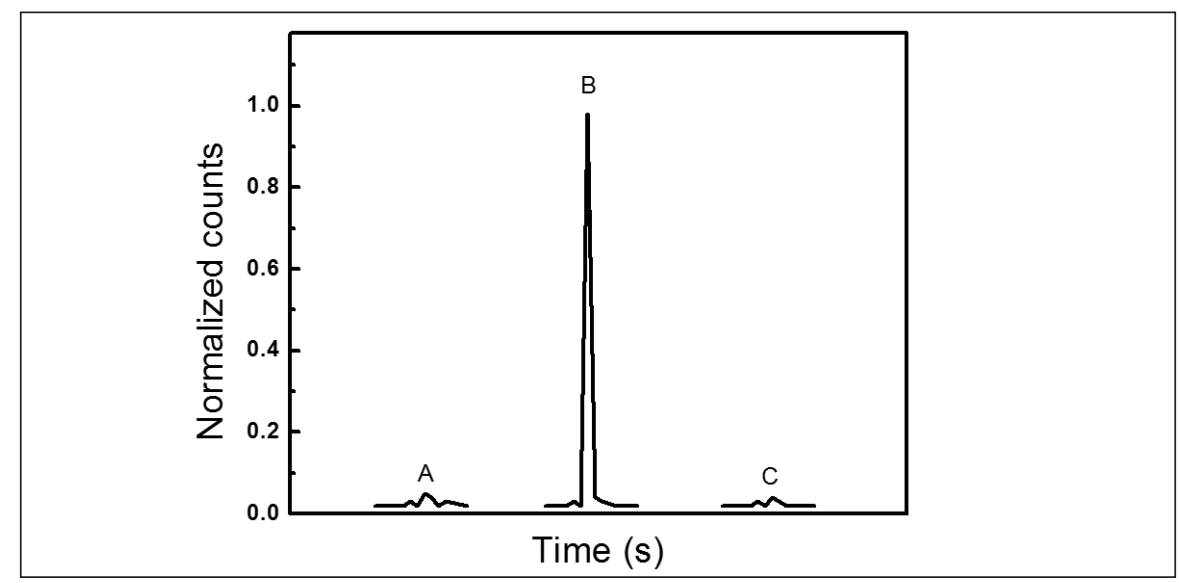

Fig. 1. Signal profiles for Y vaporized at $1200{ }^{\circ} \mathrm{C}$.

A: only PAN in 1-undecanol and THF; B: Y with PAN in 1-undecanol and THF; C: only $Y$ standard solution. Y: $5.0 \mathrm{ng}^{\mathrm{mL}} \mathrm{L}^{-1}$ 


\section{Effect of pH}

In the SFODME process, one of the critical variables for the formation of the REE-PAN complex is the sample $\mathrm{pH}$, since it directly affects the existing form of metal ions and chelating reagent. Thus, the effects of sample $\mathrm{pH}$ on the extraction efficiency of REE ions were investigated in the $\mathrm{pH}$ range of 3.0-9.0. It can be seen in Figure 2 that the signal intensities of the REEs increased rapidly with an increase in $\mathrm{pH}$ from 3.0 to 6.0 , and kept nearly constant in the $\mathrm{pH}$ range of 6.0 to 8.0 , then decreased with a further increase in $\mathrm{pH}$ from 8.0 to 9.0. Thus, $\mathrm{pH} 7.0$ was selected for subsequent experiments.

\section{Optimization of PAN Concentration}

To obtain a quantitative REE extraction from an aqueous sample and high analysis sensitivity, the PAN concentration was optimized by preparing REE solutions containing PAN in the concentration range of $5.0 \times 10^{-4}-2.5 \times 10^{-2} \mathrm{~mol} \mathrm{~L}^{-1}$. The results indicate that the signal intensity of the REEs strongly depends on the concentration of PAN, and the maximum signal was recorded after the PAN concentration was more than $1.0 \times 10^{-2} \mathrm{~mol} \mathrm{~L}^{-1}$. Therefore, $1.5 \times 10^{-2} \mathrm{~mol} \mathrm{~L}^{-1}$ of PAN was used in this work.

\section{Selection of Extraction Time}

Extraction time is an important factor influencing analysis speed and extraction efficiency. For this reason, the effect of extraction time on the signal intensities of the analytes was investigated within the time span of 5 to 60 minutes. The results showed that the signal intensities of the analytes were increased first with an increase in the extraction time, and then reached a plateau after 30 minutes. Thus, an extraction time of 30 minutes was used in the following experiments.

\section{Effect of Stirring Rate}

Effective mixing of the aqueous and organic phase is essential for the mass-transfer of the analytes from the aqueous phase to the organic phase. Thus, the effect of stirring rate on extraction was studied in the range of 400-1200 rpm. The experimental results show that the signal intensities of the analytes increased rapidly by increasing the stirring rate from 400 to $800 \mathrm{rpm}$. However, an increase in the rate of the signal intensity decreased obviously when the stirring rate exceeded $1000 \mathrm{rpm}$ owing to the loss of a floating solvent drop. In this work, a stirring rate of $800 \mathrm{rpm}$ was employed for the analysis.

\section{Effect of Sample Volume}

An important parameter which influences the preconcentration factor is sample volume. On one hand, an increase in the volume ratio of the aqueous phase to the organic phase can result in an increase of the enrichment factor. On the other hand, an increase in sample volume may lead to a decrease in extraction efficiency in a given time. Accordingly, the effect of sample solution volume on the extraction of $0.1 \mathrm{ng}$ of REE ions in different sample volumes ranging from 5.0-35 mL was investigated (Figure 3). The results indicate that when the sample volumes are lower than $20 \mathrm{~mL}$, the signal intensities of the analytes were at a maximum and remained constant, whereas a decrease of signal intensities was observed with the continuous increase of sample volume. Considering the analysis time and enrichment factor, a $10 \mathrm{~mL}$ sample volume was used for the analysis of the real samples. As described in the previous section, the analytes were quantitatively extracted using a volume of $20 \mu \mathrm{L}$ solvent drop. Thus, an enrichment factor of 500 was obtained for REE ions in the present work.

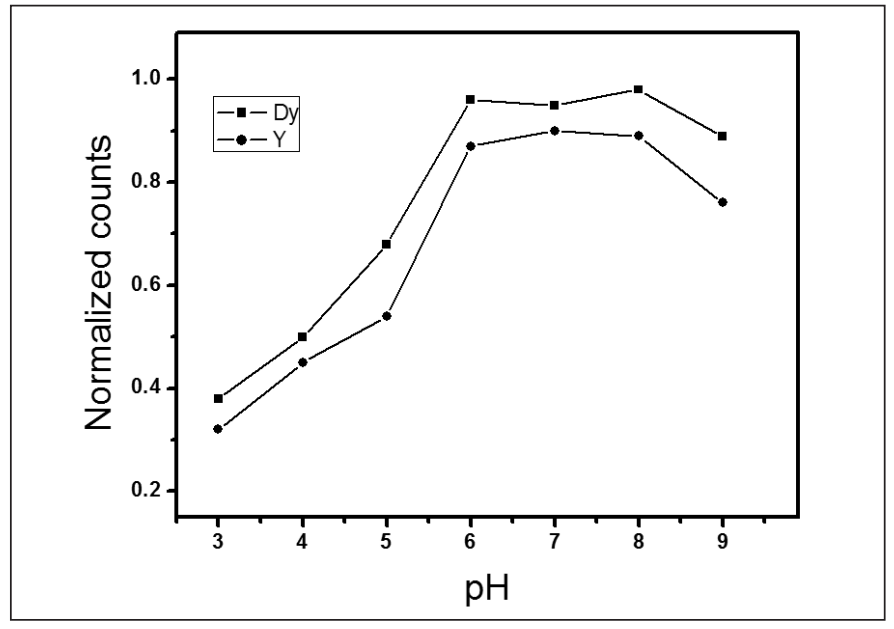

Fig. 2. Effect of $p H$ on SFODME. Dy and Y: $1.0 \mathrm{ng} m L^{-1}$

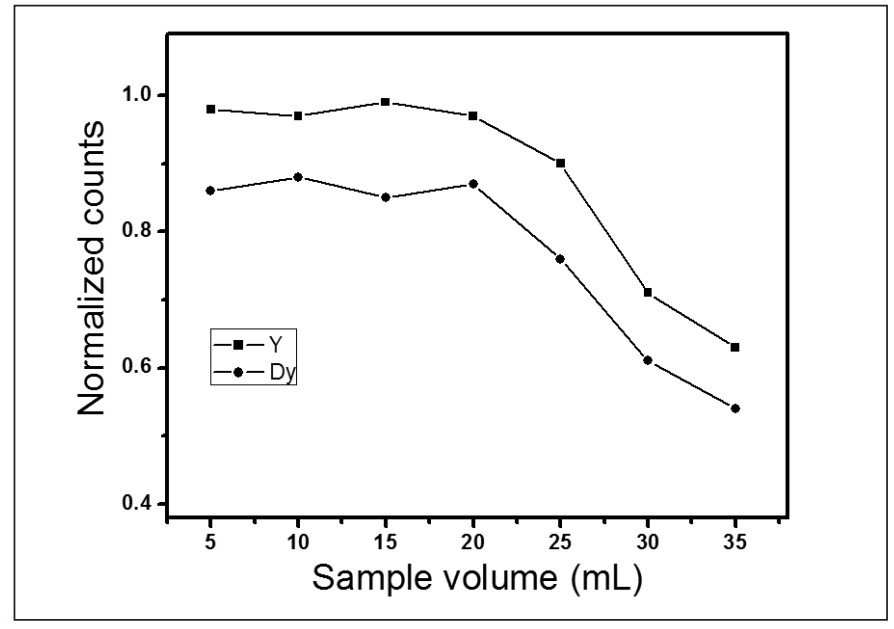

Fig. 3. Effect of sample solution volume on SFODME. $Y$ and $D y: 1.0 \mathrm{ng} \mathrm{mL}^{-1}$ 


\section{Influence of Diverse Ions}

The extraction efficiency of the method for REEs in the presence of various cations and anions was examined. The tolerance limit of potentially interfering ions, which gives less than $10 \%$ error for the determination of REEs, was evaluated. The results in Table II show that no significant interferences were observed at the given level. It is worth noting that the interfering ions such as $\mathrm{Zn}^{2+}, \mathrm{Cu}^{2+}$, and $\mathrm{Fe}^{3+}$ can also react with the PAN and then enter a competitive reaction with the REEs. Therefore, the excessive chelating reagent was necessary for this experiment.

\section{Choice of Temperature Program}

During the drying step, a drying temperature of $100^{\circ} \mathrm{C}$ and a drying time of 10 seconds were used for removal of the water vapor. The effects of charring temperature and

TABLE II

Effect of Coexisting Ions

\begin{tabular}{lr}
\hline Coexisting Ion & Conc. Ratio \\
\hline $\mathrm{Na}^{+}, \mathrm{K}^{+}$ & 10000 \\
$\mathrm{Ca}^{2+}, \mathrm{Mg}^{2+}$ & 5000 \\
$\mathrm{Fe}^{3+}, \mathrm{Zn}^{2+}, \mathrm{Al}^{3+}, \mathrm{Cu}^{2+}$ & 500 \\
$\mathrm{SO}_{4}^{2-}, \mathrm{SiO}_{3}^{2-}, \mathrm{PO}_{4}^{3-}$ & 5000 \\
$\mathrm{Cl}^{-}, \mathrm{NO}_{3}^{-}$ & 10000 \\
\hline
\end{tabular}

${ }^{a}$ Foreign ion / $\mathrm{REE}^{3+}$. time on signal intensity were studied in this work. The results showed that a charring temperature of $220^{\circ} \mathrm{C}$ and a charring time of 20 seconds were optimal to remove the extraction solvent from the furnace without signal loss of the analytes at the same time. These conditions were used for subsequent tests.

The effect of vaporization temperature on the signal intensity was also studied in the temperature range of $400-1800^{\circ} \mathrm{C}$. The results showed that the signals of the analytes increased by rising the vaporization temperature from $400^{\circ} \mathrm{C}$ to $1000{ }^{\circ} \mathrm{C}$, then reached maximum and remained constant at above $1000{ }^{\circ} \mathrm{C}$. The study on the vaporization time showed that the signal intensities reached their maximum values at about 4 seconds. Thus, $1200{ }^{\circ} \mathrm{C}$ and 4 seconds were used as the vaporization temperature and vaporization time in this work, respectively.

\section{Analytical Performance}

In accordance with IUPAC recommendation, the detection limits of Dy and Y, based on three times the standard deviation of the blank solution, were 0.019 and $0.032 \mathrm{pg}$ $\mathrm{mL}^{-1}$, respectively. The linear range of this method was found from 0.01 to $10 \mathrm{ng} \mathrm{mL}^{-1}$ with a correlation coefficient better than 0.9978 .
The relative standard deviations for the nine determination of $0.1 \mathrm{ng} \mathrm{mL}^{-1}$ of Dy and $Y$ were $4.7 \%$ and $5.4 \%$, respectively.

\section{Sample Analysis}

In order to identify the accuracy of this method, a certified reference material of tea leaves (GBW 07605) and a water sample (obtained from Wuhan, China) were analyzed under the selected conditions. The obtained results are summarized in Tables III and IV, respectively. As can be seen, the determined values were in good agreement with the certified values, and the recoveries of the analytes were reasonable for trace analysis ranging from $91.3 \%$ $108 \%$.

\section{CONCLUSION}

It has been demonstrated that solidified floating organic drop microextraction (SFODME) combined with ETV-ICP-MS can be a powerful tool for the separation, preconcentration, and determination of ultra/trace REEs in biological and environmental samples. PAN and 1-undecanol were selected as a chelating reagent and an extraction solvent in SFODME, respectively. PAN also acts as a modifier in ETV. This method has the benefits of small sample requirements, simple operation, suitable for batch operations, short sample preparation
TABLE III

Analytical Results of Analytes in Natural Water Sample

\begin{tabular}{cccc}
\hline Element & $\begin{array}{c}\text { Added } \\
\left(\mathrm{ng} \mathrm{mL} \mathrm{mL}^{-1}\right)\end{array}$ & \multicolumn{1}{c}{$\begin{array}{c}\text { Found }^{\mathrm{a}} \\
\left(\mathrm{ng} \mathrm{mL}^{-1}\right)\end{array}$} & $\begin{array}{c}\text { Recovery } \\
(\%)\end{array}$ \\
\hline Dy & 0 & $0.037 \pm 0.0031$ & - \\
& 0.1 & $0.145 \pm 0.012$ & 108.0 \\
& 0.2 & $0.232 \pm 0.015$ & 97.5 \\
$\mathrm{Y}$ & 0 & $0.183 \pm 0.011$ & - \\
& 0.5 & $0.675 \pm 0.042$ & 98.4 \\
& 1.0 & $1.15 \pm 0.085$ & 96.7 \\
\hline
\end{tabular}

\footnotetext{
${ }^{\mathrm{a}}$ Mean value \pm Standard deviation, $\mathrm{n}=3$.
}

TABLE IV

Analytical Results of Analytes in Certified Reference Material

\begin{tabular}{ccccc}
\hline Element & $\begin{array}{c}\text { Added } \\
\left(\mu \mathrm{g} \mathrm{g}^{-1}\right)\end{array}$ & $\begin{array}{c}\text { Found } \\
\left(\mu \mathrm{g} \mathrm{g}^{-1}\right)^{\mathrm{a}}\end{array}$ & $\begin{array}{c}\text { Certified } \\
\left(\mu \mathrm{g} \mathrm{g}^{-1}\right)\end{array}$ & $\begin{array}{c}\text { Recovery } \\
(\%)\end{array}$ \\
\hline Dy & 0 & $0.021 \pm 0.0019$ & $0.017^{\mathrm{b}}$ & - \\
& 0.1 & $0.112 \pm 0.08$ & - & 91.3 \\
$\mathrm{Y}$ & 0 & $0.095 \pm 0.01$ & $0.084 \pm 0.016$ & - \\
& 0.1 & $0.199 \pm 0.014$ & - & 104.0 \\
\hline
\end{tabular}

\footnotetext{
${ }^{\mathrm{a}}$ Mean value \pm standard deviation, $\mathrm{n}=3$.

${ }^{\mathrm{b}}$ Reference value.
} 
time, minimum organic solvent consumption, high enrichment factor, and sensitive detection capability.

\section{ACKNOWLEDGMENT}

Financial support from the Nature Science Foundation and the Education Department Foundation of Hubei Province in China is gratefully acknowledged.

$\overline{\text { Received August 22, } 2013}$

\section{REFERENCES}

1. R.A. Lagad, D. Alamelu, A.K. Chaudhary, and S.K. Aggarwal, At. Spectrosc. 33(4), 109 (2012).

2. E. Orvini, M. Speziali, A. Salvini, and C. Herborg, Microchem. J. 67(1-3), 97 (2000).

3. E. Sabbioni, R. Pietra, P. Gaglione, G. Vocaturo, and G. Colombo, Sci. Total. Environ. 26, 19 (1982).

4. S. Hirano and K.T. Suzuki, Environ. Health Persp. 104, 85 (1996).

5. A. Premadas and C. R.Khorge, At. Spectrosc. 27(5), 170 (2006).

6. R.K. Malhotra, K. Satyanarayana, and G.V. Ramanaiah, At. Spectrosc. 20(3), 92 (1999).

7. S. Chen, L. Zhu, D. Lu, and X. Cheng, and X. Zhou, Microchim. Acta 169, 123 (2010).

8. F. Aydin, F. Yasar, I. Aydin, and F. Guzel, Microchem. J. 98(2), 246 (2011).

9. Y.Z. Yi, S.Y. Wu, S.J. Jiang, and A.C. Sahayam, At. Spectrosc. 34(2), 39 (2013).

10. M.A. Jeannot and F.F. Cantwell, Anal. Chem. 68(13), 2236 (1996).

11. H. Liu and P.K. Dasgupta, Anal. Chem. 68(11), 1817 (1996).

12. A. Jain and K.K. Verma, Anal. Chim. Acta 706(1), 37 (2011).

13. S. Pedersen-Bjergaard and K.E. Rasmussen, Anal. Chem. 71, 2650 (1999).

14. Q. Zhou, N. Zhao, and J. Xiao, At. Spectrosc.32(2), 62 (2011).

15. M.R.K. Zanjani, Y. Yamini, S. Shariati, and J.A. Jonsson, Anal. Chim. Acta 585(2), 286 (2007).

16. I. Lopez-Garcia, R.E. Rivas, and M. Hernandez-Cordoba, Anal. Bioanal. Chem. 396, 3097 (2010).

17. M. Ghambarian, M.R. Khalili-Zanjani, Y. Yamini, A. Esrafili, and N. Yazdanfar, Talanta 81, 197 (2010).

18. S. Dadfarnia, A.M.H. Shabani, and E. Kamranzadeh, Talanta 79, 1061 (2009).

19. J. Ma, J. Zhang, X. Du, X. Lei, and J. Li, Microchim. Acta 168, 153 (2010).
20 R.E. Rivas, I. López-García, and M. Hernández-Córdoba, Anal. Methods 2, 225 (2010).

21. S. Chen, H. Zhang, and B. Ruan, At. Spectrosc. 30(4), 143 (2009). 